\title{
On Fitness in Structured Metapopulations
}

Mats Gyllenberg (matsgyl@utu.fi)

J.A.J.Metz (metz@rulsfb.leidenuniv.nl)

\section{Approved by}

Gordon J. MacDonald (macdon@iiasa.ac.at)

Director, IIASA

August 1999

Interim Reports on work of the International Institute for Applied Systems Analysis receive only limited review. Views or opinions expressed herein do not necessarily represent those of the Institute, its National Member Organizations, or other organizations supporting the work. 


\section{IIASA STUDIES IN ADAPTIVE DYNAMICS No. 37}

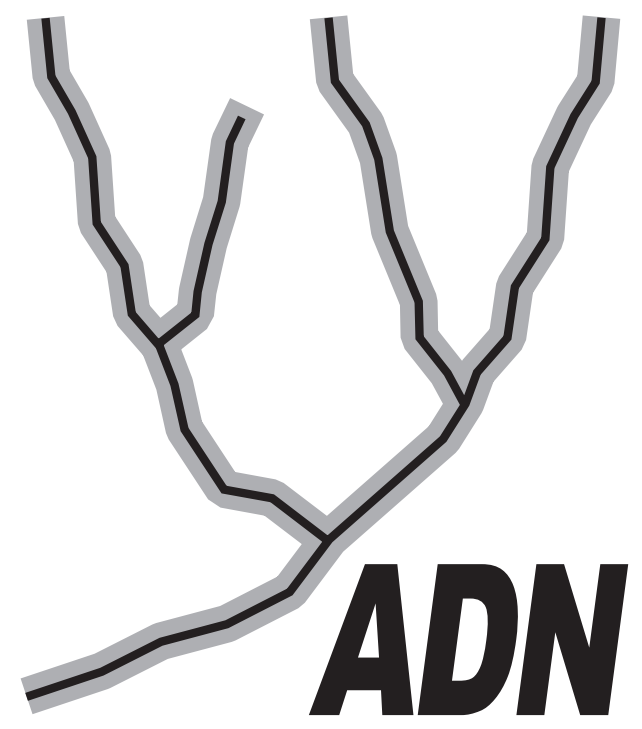

The Adaptive Dynamics Network at IIASA fosters the development of new mathematical and conceptual techniques for understanding the evolution of complex adaptive systems.

Focusing on these long-term implications of adaptive processes in systems of limited growth, the Adaptive Dynamics Network brings together scientists and institutions from around the world with IIASA acting as the central node.

Scientific progress within the network is reported in the IIASA Studies in Adaptive Dynamics series.

\section{THE ADAPTIVE DYNAMICS NETWORK}

The pivotal role of evolutionary theory in life sciences derives from its capability to provide causal explanations for phenomena that are highly improbable in the physicochemical sense. Yet, until recently, many facts in biology could not be accounted for in the light of evolution. Just as physicists for a long time ignored the presence of chaos, these phenomena were basically not perceived by biologists.

Two examples illustrate this assertion. Although Darwin's publication of "The Origin of Species" sparked off the whole evolutionary revolution, oddly enough, the population genetic framework underlying the modern synthesis holds no clues to speciation events. A second illustration is the more recently appreciated issue of jump increases in biological complexity that result from the aggregation of individuals into mutualistic wholes.

These and many more problems possess a common source: the interactions of individuals are bound to change the environments these individuals live in. By closing the feedback loop in the evolutionary explanation, a new mathematical theory of the evolution of complex adaptive systems arises. It is this general theoretical option that lies at the core of the emerging field of adaptive dynamics. In consequence a major promise of adaptive dynamics studies is to elucidate the long-term effects of the interactions between ecological and evolutionary processes.

A commitment to interfacing the theory with empirical applications is necessary both for validation and for management problems. For example, empirical evidence indicates that to control pests and diseases or to achieve sustainable harvesting of renewable resources evolutionary deliberation is already crucial on the time scale of two decades.

The Adaptive Dynamics Network has as its primary objective the development of mathematical tools for the analysis of adaptive systems inside and outside the biological realm. 


\section{IIASA STUDIES IN ADAPTIVE DYNAMICS}

No. 1 Metz JAJ, Geritz SAH, Meszéna G, Jacobs FJA, van Heerwaarden JS:

Adaptive Dynamics: A Geometrical Study of the Consequences of Nearly Faithful Reproduction.

IIASA Working Paper WP-95-099.

In: van Strien SJ, Verduyn Lunel SM (eds.): Stochastic and Spatial Structures of Dynamical Systems, Proceedings of the Royal Dutch Academy of Science (KNAW Verhandelingen), North Holland, Amsterdam, pp. 183-231 (1996).

No. 2 Dieckmann U, Law R:

The Dynamical Theory of Coevolution: A Derivation from Stochastic

Ecological Processes.

IIASA Working Paper WP-96-001.

Journal of Mathematical Biology (1996) 34, 579-612.

No. 3 Dieckmann U, Marrow P, Law R:

Evolutionary Cycling of Predator-Prey Interactions: Population Dynamics and the Red Queen.

Journal of Theoretical Biology (1995) 176, 91-102.

No. 4 Marrow P, Dieckmann U, Law R:

Evolutionary Dynamics of Predator-Prey Systems: An Ecological

Perspective.

IIASA Working Paper WP-96-002.

Journal of Mathematical Biology (1996) 34, 556-578.

No. 5 Law R, Marrow P, Dieckmann U:

On Evolution under Asymmetric Competition.

IIASA Working Paper WP-96-003.

Evolutionary Ecology (1997) 11, 485-501.

No. 6 Metz JAJ, Mylius SD, Diekmann O:

When Does Evolution Optimise? On the Relation between Types of Density

Dependence and Evolutionarily Stable Life History Parameters.

IIASA Working Paper WP-96-004.

No. 7 Ferrière R, Gatto $\mathrm{M}$ :

Lyapunov Exponents and the Mathematics of Invasion in Oscillatory or Chaotic Populations.

Theoretical Population Biology (1995) 48, 126-171.

No. 8 Ferrière R, Fox GA:

Chaos and Evolution.

Trends in Ecology and Evolution (1995) 10, 480-485.

No. 9 Ferrière R, Michod RE:

The Evolution of Cooperation in Spatially Heterogeneous Populations.

IIASA Working Paper WP-96-029.

American Naturalist (1996) 147, 692-717. 
No. 10 Van Dooren TJM, Metz JAJ:

Delayed Maturation in Temporally Structured Populations with Non-Equilibrium Dynamics.

IIASA Working Paper WP-96-070.

Journal of Evolutionary Biology (1998) 11, 41-62.

No. 11 Geritz SAH, Metz JAJ, Kisdi E, Meszéna G:

The Dynamics of Adaptation and Evolutionary Branching.

IIASA Working Paper WP-96-077.

Physical Review Letters (1997) 78, 2024-2027.

No. 12 Geritz SAH, Kisdi E, Meszéna G, Metz JAJ:

Evolutionarily Singular Strategies and the Adaptive Growth and Branching of the Evolutionary Tree.

IIASA Working Paper WP-96-114.

Evolutionary Ecology (1998) 12, 35-57.

No. 13 Heino M, Metz JAJ, Kaitala V:

Evolution of Mixed Maturation Strategies in Semelparous Life-Histories: the Crucial Role of Dimensionality of Feedback Environment.

IIASA Working Paper WP-96-126.

Philosophical Transactions of the Royal Society of London Series B (1997) 352, 16471655.

No. 14 Dieckmann U:

Can Adaptive Dynamics Invade?

IIASA Working Paper WP-96-152.

Trends in Ecology and Evolution (1997) 12, 128-131.

No. 15 Meszéna G, Czibula I, Geritz SAH:

Adaptive Dynamics in a Two-Patch Environment: a Simple Model for

Allopatric and Parapatric Speciation.

IIASA Interim Report IR-97-001.

Journal of Biological Systems (1997) 5, 265-284.

No. 16 Heino M, Metz JAJ, Kaitala V:

The Enigma of Frequency-Dependent Selection.

IIASA Interim Report IR-97-061.

Trends in Ecology and Evolution (1998) 13, 367-370.

No. 17 Heino M:

Management of Evolving Fish Stocks.

IIASA Interim Report IR-97-062.

Canadian Journal of Fisheries and Aquatic Sciences (1998) 55, 1971-1982.

No. 18 Heino M:

Evolution of Mixed Reproductive Strategies in Simple Life-History Models.

IIASA Interim Report IR-97-063.

No. 19 Geritz SAH, van der Meijden E, Metz JAJ:

Evolutionary Dynamics of Seed Size and Seedling Competitive Ability.

IIASA Interim Report IR-97-071.

Theoretical Population Biology (1999) 55, 324-343. 
No. 20 Galis F, Metz JAJ:

Why are there so many Cichlid Species? On the Interplay of Speciation and Adaptive Radiation.

IIASA Interim Report IR-97-072.

Trends in Ecology and Evolution (1998) 13, 1-2.

No. 21 Boerlijst MC, Nowak MA, Sigmund K:

Equal Pay for all Prisoners. /The Logic of Contrition.

IIASA Interim Report IR-97-073.

AMS Monthly (1997) 104, 303-307.

Journal of Theoretical Biology (1997) 185, 281-294.

No. 22 Law R, Dieckmann U:

Symbiosis without Mutualism and the Merger of Lineages in Evolution.

IIASA Interim Report IR-97-074.

Proceedings of the Royal Society of London Series B (1998) 265, 1245-1253.

No. 23 Klinkhamer PGL, de Jong TJ, Metz JAJ:

Sex and Size in Cosexual Plants.

IIASA Interim Report IR-97-078.

Trends in Ecology and Evolution (1997) 12, 260-265.

No. 24 Fontana W, Schuster P:

Shaping Space: The Possible and the Attainable in RNA Genotype-Phenotype Mapping.

IIASA Interim Report IR-98-004.

Journal of Theoretical Biology (1998) 194, 491-515.

No. 25 Kisdi E, Geritz SAH:

Adaptive Dynamics in Allele Space: Evolution of Genetic Polymorphism by Small Mutations in a Heterogeneous Environment.

IIASA Interim Report IR-98-038.

No. 26 Fontana W, Schuster P:

Continuity in Evolution: On the Nature of Transitions.

IIASA Interim Report IR-98-039.

Science (1998) 280, 1451-1455.

No. 27 Nowak MA, Sigmund K:

Evolution of Indirect Reciprocity by Image Scoring. / The Dynamics of Indirect Reciprocity.

IIASA Interim Report IR-98-040.

Nature (1998) 393, 573-577.

Journal of Theoretical Biology (1998) 194, 561-574.

No. 28 Kisdi E:

Evolutionary Branching Under Asymmetric Competition.

IIASA Interim Report IR-98-045.

Journal of Theoretical Biology (1999) 197, 149-162.

No. 29 Berger U:

Best Response Adaptation for Role Games.

IIASA Interim Report IR-98-086. 
No. 30 Van Dooren TJM:

The Evolutionary Ecology of Dominance-Recessivity

IIASA Interim Report IR-98-096.

Journal of Theoretical Biology (1999) 198, 519-532.

No. 31 Dieckmann U, O'Hara B, Weisser W:

The Evolutionary Ecology of Dispersal.

IIASA Interim Report IR-98-108.

Trends in Ecology and Evolution (1999) 14, 88-90.

No. 32 Sigmund K:

Complex Adaptive Systems and the Evolution of Reciprocation.

IIASA Interim Report IR-98-100.

Ecosystems (1998) 1, 444-448.

No. 33 Posch M, Pichler A, Sigmund K:

The Efficiency of Adapting Aspiration Levels.

IIASA Interim Report IR-98-103.

Proceedings of the Royal Society of London Series B (1999) 266, 1427-1435.

No. 34 Mathias A, Kisdi É:

Evolutionary Branching and Coexistence of Germination Strategies.

IIASA Interim Report IR-99-014.

No. 35 Dieckmann U, Doebeli M:

On the Origin of Species by Sympatric Speciation.

IIASA Interim Report IR-99-013.

Nature (1999) 400, 354-357.

No. 36 Metz JAJ, Gyllenberg M:

How Should We Define Fitness in Structured Metapopulation Models? Including an Application to the Calculation of Evolutionarily Stable Dispersal Strategies.

IIASA Interim Report IR-99-019.

No. 37 Gyllenberg M, Metz JAJ:

On Fitness in Structured Metapopulations.

IIASA Interim Report IR-99-037.

Issues of the IIASA Studies in Adaptive Dynamics series can be obtained free of charge. Please contact:

Adaptive Dynamics Network

International Institute for Applied Systems Analysis

Schlossplatz 1

A-2361 Laxenburg

Austria

Telephone +432236 807, Telefax +432236 71313, E-Mail adn@iiasa.ac.at, Internet http://www.iiasa.ac.at/Research/ADN 


\section{Contents}

1 Introduction $\quad 1$

2 Structured metapopulation dynamics 2

3 A class of structured metapopulation models 4

4 The fitness of a rare mutant 5

$\begin{array}{lll}5 & \text { An example } & 7\end{array}$ 


\begin{abstract}
In this paper we present a mathematical definition of fitness in general structured metapopulation models. We apply the theory to a model structured by local population size and in which local dynamics is explicitly modelled. In particular, we calculate the evolutionarily stable dispersal strategy in the case where only dispersal is subject to evolutionary control but all other model ingredients are assumed fixed. In this case we show that there exists a threshold size such that at ESS everyone should stay as long as the population size is below the threshold and everyone should disperse immediately as the population size reaches the threshold.
\end{abstract}




\title{
About the Authors
}

\author{
Mats Gyllenberg \\ Department of Mathematics \\ University of Turku \\ FIN-20014 Turku, Finland \\ J.A.J. Metz \\ Institute for Evolutionary and Ecological Sciences \\ Leiden University \\ NL-2311 GP Leiden \\ The Netherlands \\ and \\ Adaptive Dynamics Network \\ International Institute for Applied Systems Analysis \\ A-2361 Laxenburg, Austria
}

\section{Acknowledgment}

The work of Mats Gyllenberg was supported by the Academy of Finland. Two workshops at Tvärminne Zoological Station, Finland have been crucial for paper: The workshop "Evolution of Dispersal" organized by the Spatial Ecology Program at the Division of Population Biology, University of Helsinki, October 15 -18, 1998, and the ESF workshop "Metapopulation Dynamics", April 15-18, 1999. 


\title{
On Fitness in Structured Metapopulations
}

\author{
Mats Gyllenberg \\ J.A.J. Metz
}

\section{Introduction}

During the past decade we have witnessed an increasing interest in the dynamics of metapopulations. The book edited by Hanski and Gilpin (1997) and the book by Hanski (1999) contain more than 1000 references each. At the same time the evolution of dispersal has caught the interest of many scientists (Hastings 1983; Holt and McPeek 1996; Doebeli and Ruxton 1997; Parvinen 1999). Long-term evolution is the result of invasions of mutant traits and the success of invasion attempts is determined by the fitness of the mutant. As most species have a hierarchical spatial structure with several local populations connected by dispersal comprising a metapopulation, the evolution of migration or dispersal is most conveniently modelled in the framework of metapopulation dynamics. It is the purpose of the present paper to present a mathematical definition of fitness in structured metapopulation models.

In a single population fitness is usually defined as the long-term exponential growth rate $r(E)$ of a phenotype in a given environment $E$ (Metz, Nisbet and Geritz 1992). Here one should think of the environment as an interaction variable through which all (nonlinear) feedback takes place. If the environment is constant, $E(t) \equiv \bar{E}$, then one can alternatively and equivalently use the basic reproduction number $R(\bar{E})$ as fitness measure because it is well known that $R(\bar{E})$ is less than, equal to, or greater than 1 depending on whether $r(\bar{E})$ is less than, equal to, or greater than 0 . $R(\bar{E})$ is the expected lifetime production of offspring. The difference between $r$ and $R$ is that $r$ is the growth rate in real time, whereas $R$ operates at the generation level.

The question concerning successful invasion is easily addressed using the basic reproduction number $R$. Assume that the resident population is in demographical equilibrium corresponding to a constant environment $\bar{E}^{\text {res }}$. Since at demographical equilibrium every individual on average exactly replaces itself one has $R^{\text {res }}\left(\bar{E}^{\text {res }}\right)=$ 1. The basic reproduction number $R$ depends on both the environment and the strategy and the superscript res refers to the strategy played by the resident. A mutant playing a different strategy can invade if and only if

$$
R^{\text {mut }}\left(\bar{E}^{\text {res }}\right)>1
$$

This means that a mutant can invade if and only if its basic reproduction number in the environment set by the resident is greater than 1 . 
When we want to apply the procedure outlined above to metapopulations we encounter several difficulties. The most obvious one is that even if the environmental interaction variable is constant at the resident attractor, a mutant experiences different conditions in different patches, and moreover, the conditions change in the patch due to the development of the resident local population. So it is far from obvious how to define $R^{\text {mut }}\left(\bar{E}^{\text {res }}\right)$ in the case of metapopulation models.

In this paper we present a mathematical definition of $R^{\text {mut }}\left(\bar{E}^{\text {res }}\right)$ for a large class of structured metapopulation models including models with stochasticity at the level of local populations. In Section 5 we give an explicit formula for $R^{\text {mut }}\left(\bar{E}^{\text {res }}\right)$ for a model with deterministic growth of local populations. We also calculate the evolutionarily stable dispersal strategy assuming that the tendency to migrate depends only upon the local population size and not for instance on the age of the individual. Because of the deterministic nature of local dynamics this model assumes infinite local populations. In a companion paper (Metz and Gyllenberg 1999) we work out the details for the finite local population case and give recepies to efficiently calculate $R^{\text {mut }}\left(\bar{E}^{\text {res }}\right)$ using existing software. In that paper we also calculate the evolutionarily stable dispersal strategy for the case of juvenile migration for the case of infinite local populations as opposed to the age-independent migration considered in the present paper.

\section{Structured metapopulation dynamics}

In this paper we model structured metapopulations in the spirit of Gyllenberg, Hanski and Hastings (1997). We start by giving a brief description of that part of the theory which is essential for our present needs. As pointed out by Metz and Diekmann (1986) (see also Diekmann et al. 1988, 1989), the theory of structured populations can be applied to metapopulations in a rather straightforward manner if one makes the analogy between local populations and individuals and between metapopulation and population. Our approach is therefore merely an adaptation of the general structured population framework of Diekmann, Gyllenberg, Metz and Thieme $(1993,1998)$ to metapopulation models.

We regard a metapopulation as a population of populations or, more generally, as a population of local entities. In addition to local populations, local entities can represent patches, dispersers etc. A local entity is characterized by its state $x$, which typically is a vector in a finite dimensional space. The components of $x$ may for instance stand for patch quality or area or size of a local population.

A local entity develops (i.e., its state changes with time) as a consequence of for instance patch quality dynamics, local population growth due to births, deaths and migration; it gives rise to new local entities (e.g. local populations produce dispersers, dispersers colonize empty patches); and vanishes (e.g. when a local population goes extinct or a patch is destroyed). To model mechanisms at the local level, we therefore need two ingredients, one describing the production of new local entities and one describing the development and survival of local entities.

In this paper we shall only be concerned with constant environments and this makes the notation much simpler as compared with the one in (Gyllenberg, Hanski and Hastings 1997). In particular we shall drop the overbar on constant environ- 
ments and since the environment is always assumed to be set by th resident, the superscript res becomes superfluous. We thus write simply $E$ instead of $\bar{E}^{\text {res }}$.

We let $\Omega$ denote the local state space, that is, the set of all admissible local states. We introduce the lifetime cumulative reproduction measure $\Lambda$ and the local development measure as follows: For each given constant environment $E$, each $x \in \Omega$ and each measurable set $\omega \subset \Omega$ we let

$$
\begin{aligned}
\Lambda_{E}(x)(\omega)= & \text { expected number of new local entities with birth state in } \omega \\
& \text { produced by a local entity with birth state } x \text { during its entire } \\
& \text { life, } \\
u_{E}(x ; t)(\omega)= & \text { probability that a local entity with state } x \text { will still be alive and } \\
& \text { have state in } \omega t \text { time units later. }
\end{aligned}
$$

The measures $\Lambda_{E}(x)$ and $u_{E}(x ; t)$ are not independent but satisfy certain consistency relations (Diekmann et al. 1998; Gyllenberg et al. 1997). For instance, the measures $u_{E}(x ; t)$ are the transition probabilities of a Markov process and therefore they satisfy the Chapman-Kolmogorov relation.

The metapopulation state is by definition the distribution of local states and is represented by a measure $m \in M_{+}(\Omega)$, the set of all finite positive Borel measures in $\Omega$. We can now lift the model to the metapopulation level by defining the next generation operator $W$ and the next state operators $T(t), t \geq 0$ acting on $M_{+}(\Omega)$. For each given constant environment $E$, each $m \in M_{+}(\Omega)$ and each measurable set $\omega \in \Omega$ we set

$$
\begin{aligned}
\left(W_{E} m\right)(\omega) & =\int_{\Omega} \Lambda_{E}(x)(\omega) m(d x) \\
\left(T_{E}(t) m\right)(\omega) & =\int_{\Omega} u_{E}(x ; t)(\omega) m(d x) .
\end{aligned}
$$

$\left(W_{E} m\right)(\omega)$ is the expected number of new local entities with birth state in $\omega$ produced by a collection of local entities distributed as $m$ during their entire lives. $T_{E}(t) m$ is the distribution at time $t$ of a collection of local entities which at time zero were distributed as $m$. It follows from the fact that $u_{E}$ satisfies the ChapmanKolmogorov relation that $\left\{T_{E}(t)\right\}_{t \geq 0}$ is a semigroup.

Following Diekmann et al. (1990, 1998), Heesterbeek (1992) and Gyllenberg et al. (1997) we now define the basic reproduction number $R(E)$ as the spectral radius of the operator $W_{E}$.

In most cases of interest positivity arguments guarantee that $R(E)$ is an eigenvalue and that all other eigenvalues have absolute value less than or equal to $R(E)$. Let $b_{E}$ be the eigenvector corresponding to $R(E) . \quad b_{E}$ and $R(E)$ have important biological interpretations: $b_{E}$ is the distribution of birth states at equilibrium and $R(E)$ is the expected number of new local entities produced by one "typical", that is, sampled from $b_{E}$, local entity during its entire life.

Usually the set $\Omega_{b}$ of admissible birth states is much smaller than the local state space $\Omega$. It is clear that for solving the eigenvalue problem (determining $R(E)$ ) one only has to consider the restriction of $W_{E}$ to $M\left(\Omega_{b}\right)$. In many models there are only a finite number of admissible birth states. When this is the case determining $R(E)$ boils down to finding the dominant eigenvalue of a nonnegative (finite dimensional) 
matrix. Without risk of confusion we shall use the same symbol for $W_{E}$ and its restriction to $M\left(\Omega_{b}\right)$ or the corresponding matrix.

The steady metapopulation state corresponding to $E$ is given by

$$
m_{E}=\int_{0}^{\infty} T_{E}(t) b_{E} d t
$$

A necessary condition for equilibrium is

$$
R(E)=1 \text {. }
$$

Often, and in particular in the cases considered below, the environmental interaction variable $E$ is connected to the metapopulation state through a linear operator $A$ and this gives us an additional equilibrium condition:

$$
E=A m_{E}
$$

We emphasize that at the local level the model is stochastic and that it therefore allows for finite local population sizes taking on integer values. But the way in which the model is lifted to the metapopulation level involves taking expectations and therefore the full model is deterministic and based on the tacit assumption of an infinite number of patches.

\section{A class of structured metapopulation models}

In this section we specify the class of models to which the general framework of Section 2 will be applied.

We shall assume that there are two types of local entities: local populations and dispersers. We assume that selection operates on the level of individuals and therefore we shall always include local population size $x_{1}$ as a component of the structuring variable $x$ of local populations. Other components can for instance reflect patch quality and area (Hanski and Gyllenberg 1993, 1997; Gyllenberg and Hanski 1997) and they may or may not be dynamical variables. The set of all admissible $x$ is denoted by $\Omega_{p}$ ( $p$ for population). The dispersers are unstructured and they do not reproduce during migration. The condition of being a disperser is symbolically represented by " $d$ ". The local state space is thus $\Omega=\Omega_{p} \cup\{d\}$. The total patch density will be scaled to 1 .

The density $D$ of dispersers affects local dynamics through immigration. This is most conveniently modelled by taking $D$ as one component of the environmental interaction variable (Gyllenberg and Hanski 1992). We shall do so in this paper.

We shall allow for local disasters in which all individuals of a patch die. A local disaster is considered as the simultaneous death of the old local population and the birth of a new local population with size zero. This is a valid model assumption, since in our deterministic setting there is a continuous inflow of migrants from the disperser pool: There are no empty patches.

Emigration is interpreted as a local population giving birth to a disperser. The set of population birth states is therefore $\Omega_{b}=\Omega_{b p} \cup\{d\}$, where $\Omega_{b p}$ is a subset of $\left\{x \in \Omega_{p} \mid x_{1}=0\right\}$. 
We represent a measure on $\Omega$ by a 2 -vector the first component of which is a measure $p$ on $\Omega_{p}$ and the second component is a real number $D$. Because there are no empty patches in our model, $p$ is a probability measure, that is, its total mass equals 1 . Similarly a measure on $\Omega_{b}$ is represented by a vector with components consisting of a measure $b^{(p)}$ on $\Omega_{b p}$ and a real number $b^{(d)}$. The restriction of $\Lambda_{E}(x)$ to $\Omega_{b p}$ is denoted by $\Lambda_{E}^{(p)}(x)$ and the restriction to $\{d\}$ is denoted by $\Lambda_{E}^{(d)}(x) . \Lambda_{E}^{(d)}(x)$ is simply the expected number of dispersers produced by a local population born in state $x$ during its lifetime. By our model description we have

$$
W_{E}\left(\begin{array}{c}
b^{(p)} \\
b^{(d)}
\end{array}\right)=\left(\begin{array}{cc}
W_{E}^{(p)} & 0 \\
W_{E}^{(d)} & 0
\end{array}\right)\left(\begin{array}{c}
b^{(p)} \\
b^{(d)}
\end{array}\right)=\left(\begin{array}{c}
\int_{\Omega_{b p}} \Lambda_{E}^{(p)}(x) b^{(p)}(d x) \\
\int_{\Omega_{b p}} \Lambda_{E}^{(d)}(x) b^{(p)}(d x)
\end{array}\right)
$$

Because by our model assumption there is neither loss nor gain of local populations it is clear that the spectral radius of $W_{E}^{(p)}$ is one and hence the same is true of $W_{E}$. It follows that

$$
R(E)=1 \text {. }
$$

The eigenvector $b_{E}=\left(b_{E}^{(p)}, b_{E}^{(d)}\right)^{T}$ corresponding to $R(E)=1$ is now obtained by first solving

$$
\int_{\Omega_{b p}} \Lambda_{E}^{(p)}(x) b_{E}^{(p)}(d x)=b_{E}^{(p)}
$$

and then defining

$$
b_{E}^{(d)}=\int_{\Omega_{b p}} \Lambda_{E}^{(d)}(x) b_{E}^{(p)}(d x) .
$$

Finally the equilibrium metapopulation state is obtained from (2.3) and (2.2)

$$
\begin{aligned}
p_{E} & =\int_{0}^{\infty} \int_{\Omega_{b p}} u_{E}(x ; t) b_{E}^{(p)}(d x) d t \\
D & =\tau b_{E}^{(d)}
\end{aligned}
$$

where

$$
\tau=\int_{0}^{\infty} u_{E}(d ; t) d t
$$

is the expected time a disperser stays (until dying or immigrating into a patch) in the disperser pool.

Note that in the calculations above we have pretended that the constant environment $E$ is given. As we have pointed out $E$ is determined by the metapopulation state and contains $D$ as a component. Therefore (3.5) and (3.6) are not explicit formulas but equations from which $E$ (and hence $D$ ) can be solved. An example of how this can be done is given in Section 5 .

\section{The fitness of a rare mutant}

Assume that the resident population has reached an equilibrium $m_{E}$ corresponding to a constant environment $E$. The fitness of a rare mutant is in principle defined in a straightforward manner along the lines outlined in the introduction and in Section 
2. We thus let $\Lambda_{E}^{\text {mut }}$ be the lifetime cumulative reproduction measure of mutants when the environment is set by the resident. The next generation operator of the mutant is then analogously with (2.1) given by

$$
\left(W_{E}^{\mathrm{mut}} m\right)(\omega)=\int_{\Omega} \Lambda_{E}^{\mathrm{mut}}(x)(\omega) m(d x)
$$

and $R^{\text {mut }}(E)$ is defined as the spectral radius of $W_{E}^{\text {mut }}$.

When it comes to the actual specification of the reproduction measures we observe a fundamental difference between $\Lambda_{E}^{\mathrm{mut}}$ and $\Lambda_{E}^{\mathrm{res}}$. Recall from Section 3 that there is no reproduction event associated with a resident disperser immigrating into a patch - the local resident population already exists and immigration will only affect the growth of the local population and not its existence. For the mutant the situation is different. A mutant disperser arriving at a patch with only resident individuals will indeed initiate a new mutant local population. After that the development of the mutant local population depends only on the state of the resident local population in the same patch, because the rarity of the mutant makes its contribution to density dependent effects negligible. This means that the mutant population is structured by the state of the corresponding local resident population. Contrary to the case of the resident for which the admissible birth states of local populations were restricted to a subset $\Omega_{b p}$ of $\left\{x \in \Omega_{p} \mid x_{1}=0\right\}$, the mutant local populations can have any birth state in $\Omega_{p}$.

Precisely as for the resident a mutant local population is considered to produce or "give birth" to mutant dispersers.

In order to derive a simple formula for the mutant fitness we make the additional assumption that dispersers choose their new patch at random. Let $\pi^{\text {mut }}$ be the probability that a mutant disperser survives migration. With the above assumptions we find that

$$
W_{E}^{\text {mut }}\left(\begin{array}{c}
b^{(p)} \\
b^{(d)}
\end{array}\right)=\left(\begin{array}{c}
\pi^{\mathrm{mut}} b^{(d)} p_{E} \\
\int_{\Omega_{p}} \Lambda_{E}^{(d) \mathrm{mut}}(x) b^{(p)}(d x)
\end{array}\right)
$$

The mutant fitness is now obtained as a solution to the eigenvalue problem

$$
W_{E}^{\text {mut }}\left(\begin{array}{c}
b^{(p)} \\
b^{(d)}
\end{array}\right)=R^{\text {mut }}(E)\left(\begin{array}{c}
b^{(p)} \\
b^{(d)}
\end{array}\right)
$$

It follows from (4.2) and (4.3) that

$$
R^{\mathrm{mut}}(E)=\sqrt{\pi^{\mathrm{mut}} \int_{\Omega_{p}} \Lambda_{E}^{(d) \mathrm{mut}}(x) p_{E}(d x)} .
$$

Formula (4.4) calls for some comments. First of all $-R^{\text {mut }}(E)$ is also an eigenvalue of $W_{E}^{\text {mut }}$ so the spectral radius is not a strictly dominant eigenvalue. This is due to the fact that the mutant has alternating generations: dispersers give rise to local populations and vice versa, so at the generation level the mutant metapopulation oscillates. On the other hand, because the generations overlap, there will be convergence towards a stable metapopulation state in real time.

The invasion criterion $R^{\text {mut }}(E)>1$ is of course equivalent to the condition $\left(R^{\text {mut }}(E)\right)^{2}>1$ and because the period on the generation level is two generations, the 
square of the basic reproduction number is easier to interpret biologically. Because dispersers are not assumed to reproduce (in the ordinary sense of the word) mutation always occur in local populations. Mutations can occur in any local population. The probability that the mutation occurs in a local population with state in $\omega$ is $p_{E}(\omega)$. This mutant produces a local mutant colony. The expected number of dispersers produced by such a colony is $\int_{\Omega_{p}} \Lambda_{E}^{\text {mut }(d)}(x) p_{E}(d x)$. Of these a fraction $\pi^{\text {mut }}$ will reach a habitat patch where they can found a new colony. The product of these two last mentioned numbers is $\left(R^{\text {mut }}(E)\right)^{2}$ which therefore is the expected number of new mutant colonies produced by one newly started mutant colony in an otherwise mutant free metapopulation. Thus $\left(R^{\mathrm{mut}}(E)\right)^{2}$ is here the direct localentity analogue of the usual individual level concept of basic reproduction number.

\section{An example}

In the general discussion above we have assumed that $\Lambda_{E}^{\text {mut }}$ is given beforehand. In applications the reproduction measure of mutants has, however, to be determined from the vital rates of both the resident and the mutant. In this section we shall illustrate the general theory by calculating $\left(R^{\text {mut }}(E)\right)^{2}$ for a concrete example.

We assume that the local populations are only structured by size and that the density $D$ of dispersers is the only component of the environmental interaction variable. We thus write $D=E$. The local population state space is $\Omega_{p}=[0, \infty)$. The space of admissible local population birth states of the resident is a single point: $\Omega_{b p}=\{0\}$. The birth state measure can therefore be represented by a vector $\left(b^{(p)}, b^{(d)}\right)^{T} \in \mathbf{R}^{2}$.

Our model is specified by the following ingredients:

$g(x)$ density dependent per capita growth rate due to local births and deaths,

$k(x)$ density dependent per capita emigration rate,

$\alpha$ immigration rate per disperser,

$\nu$ death rate per disperser,

$\mu(x)$ density dependent local disaster rate.

The first four of these rates describe individual behaviour. Therefore they depend on the strategy and will be equipped with superscripts res and mut whenever it is needed. The disaster rate $\mu$ operates at the level of local populations and is therefore independent of the strategy (recall that we assume that selection occurs at the individual level).

When two types are simultaneously present it is conceivable that they affect the local environment in different ways and therefore the functions $g, k$ and $\mu$ are in general functions of both the resident and mutant densities. However, below we shall only consider the invasion problem in the case of infinite local populations and 
there the mutant is present only in infinitesimal quantities so its influence on these functions can be neglected.

Local dynamics are deterministic and are governed by the ordinary differential equation

$$
\frac{d x}{d t}=g(x) x-k(x) x+\alpha D
$$

This means that we assume that local populations are infinitely large. For a discussion of how the infinite local population size case is obtained as a limit of Markov processes describing the finite population case we refer to the paper by Metz and Gyllenberg (1999).

For constant $D$ the local population size can never exceed the least positive $x$ for which the right hand side of $(5.1)$ is zero. We denote this value by $x_{\max }(D)$. If the right hand side of (5.1) is positive for all $x \in[0, \infty)$, then $x_{\max }(D)=\infty$.

For constant $D$, let $X_{D}(y ; t)$ be the solution of $(5.1)$ with initial condition $x(0)=$ $y$. Then

$$
u_{D}(x ; t)= \begin{cases}\exp \left(-\int_{0}^{t} \mu\left(X_{D}(x ; s) d s\right)\right) \delta_{X_{D}(x ; t)} & \text { if } x \in[0, \infty) \\ \exp (-(\alpha+\nu) t) \delta_{d} & \text { if } x=d .\end{cases}
$$

Here $\delta_{x}$ denotes the point mass concentrated at $x$. The expected time a disperser spends migrating is

$$
\tau=\frac{1}{\alpha+\nu}
$$

and the probability of surviving dispersal is

$$
\pi=\frac{\alpha}{\alpha+\nu}
$$

Next we derive the equilibrium of the resident metapopulation. The expected number of dispersers produced by a newly initiated local population during its entire life is

$$
\begin{aligned}
& \Lambda_{D}^{\mathrm{res}(d)}(0)=\int_{0}^{x_{\max }(D)} \frac{k^{\mathrm{res}}(x) x}{g^{\mathrm{res}}(x) x-k^{\mathrm{res}}(x) x+\alpha^{\mathrm{res}} D} \\
& \times \exp \left(-\int_{0}^{x} \frac{\mu(\xi)}{g^{\mathrm{res}}(\xi) \xi-k^{\mathrm{res}}(\xi) \xi+\alpha^{\mathrm{res}} D} d \xi\right) d x .
\end{aligned}
$$

The expected lifetime of a local population is

$$
\begin{gathered}
\ell_{D}^{\mathrm{res}}=\int_{0}^{x_{\max }(D)} \frac{1}{g^{\mathrm{res}}(x) x-k^{\mathrm{res}}(x) x+\alpha^{\mathrm{res}} D} \\
\times \exp \left(-\int_{0}^{x} \frac{\mu(\xi)}{g^{\mathrm{res}}(\xi) \xi-k^{\mathrm{res}}(\xi) \xi+\alpha^{\mathrm{res}} D} d \xi\right) d x .
\end{gathered}
$$

The eigenvalue problem takes the form

$$
W_{D}\left(\begin{array}{c}
b^{(p)} \\
b^{(d)}
\end{array}\right)=\left(\begin{array}{cc}
1 & 0 \\
\Lambda_{D}^{\mathrm{res}(d)}(0) & 0
\end{array}\right)\left(\begin{array}{l}
b^{(p)} \\
b^{(d)}
\end{array}\right) .
$$


The first component of Equation $(5.7)$ is $b_{D}^{(p)}=b_{D}^{(p)}$ and gives no information. The other component yields

$$
b_{D}^{(d)}=\Lambda_{D}^{\mathrm{res}(d)}(0) b_{D}^{(p)} .
$$

It follows from (3.5) and (5.2) that

$$
\begin{gathered}
p_{D}(d x)=\frac{b^{(p)}}{g^{\mathrm{res}}(x) x-k^{\mathrm{res}}(x) x+\alpha^{\mathrm{res}} D} \\
\times \exp \left(-\int_{0}^{x} \frac{\mu(\xi)}{g^{\mathrm{res}}(\xi) \xi-k^{\mathrm{res}}(\xi) \xi+\alpha^{\mathrm{res}} D} d \xi\right) d x .
\end{gathered}
$$

Because $p_{D}$ is a probability measure one has

$$
b_{D}^{(p)}=\frac{1}{\ell_{D}^{\text {res }}} .
$$

From (3.6), (5.3), (5.4), (5.8) and (5.10) we now deduce the equation

$$
\alpha^{\mathrm{res}} D=\pi^{\mathrm{res}} \frac{\Lambda_{D}^{\mathrm{res}(d)}(0)}{\ell_{D}^{\mathrm{res}}}
$$

from which the equilibrium number $D$ of dispersers per patch can be solved. Note that equation (5.11) is a balance equation. It says that at equilibrium the immigration rate equals the emigration rate times the probability of surviving dispersal.

Next we derive the expression for the mutant fitness. In order to apply formula (4.4) we still have to calculate $\Lambda_{D}^{\operatorname{mut}(d)}(x)$. To do so, recall that since the mutant is rare it does not affect the local population dynamics. The structuring variable $x$ (the size of the local resident population) will still grow according to (5.2). If the mutation happened in a local population of size $x$ the mutant population will therefore grow thereafter (as long at it remains rare) according to the time dependent linear ordinary differential equation

$$
\frac{d y}{d t}=\left(g^{\mathrm{mut}}\left(X_{D}(x ; t)\right)-k^{\mathrm{mut}}\left(X_{D}(x ; t)\right)\right) y .
$$

We conclude that the expected number of mutant dispersers produced by a mutant local population that was initiated when the corresponding resident local population had size $x$ is

$$
\begin{aligned}
& \Lambda_{D}^{\operatorname{mut}(d)}(x)=\int_{x}^{x_{\max }(D)} \frac{k^{\mathrm{mut}}(y)}{g^{\mathrm{res}}(y) y-k^{\mathrm{res}}(y) y+\alpha^{\mathrm{res}} D} \\
& \quad \times \exp \left(\int_{x}^{y} \frac{g^{\mathrm{mut}}(\xi)-k^{\mathrm{mut}}(\xi)-\mu(\xi)}{g^{\mathrm{res}}(\xi) \xi-k^{\mathrm{res}}(\xi) \xi+\alpha^{\mathrm{res}} D} d \xi\right) d y .
\end{aligned}
$$

Hence we obtain the following formula for the fitness of the mutant:

$$
\begin{aligned}
R^{\mathrm{mut}}(D)= & \frac{\alpha^{\mathrm{mut}}}{\alpha^{\mathrm{mut}}+\nu^{\mathrm{mut}}} \frac{1}{\ell_{D}^{\mathrm{res}}} \int_{0}^{\infty} \int_{x}^{x_{\max }(D)} \frac{k^{\mathrm{mut}}(y)}{g^{\mathrm{res}}(y) y-k^{\mathrm{res}}(y) y+\alpha^{\mathrm{res}} D} \\
& \times \exp \left(\int_{x}^{y} \frac{g^{\mathrm{mut}}(\xi)-k^{\mathrm{mut}}(\xi)-\mu(\xi)}{g^{\mathrm{res}}(\xi) \xi-k^{\mathrm{res}}(\xi) \xi+\alpha^{\mathrm{res}} D} d \xi\right) d y
\end{aligned}
$$




$$
\times \frac{1}{g^{\mathrm{res}}(x) x-k^{\mathrm{res}}(x) x+\alpha^{\mathrm{res}} D} \exp \left(-\int_{0}^{x} \frac{\mu(\xi)}{g^{\mathrm{res}}(\xi) \xi-k^{\mathrm{res}}(\xi) \xi+\alpha^{\mathrm{res}} D} d \xi\right) d x .
$$

Here $D$ is a solution of (5.11).

We close this section by calculating the evolutionarily stable dispersal strategy, when only $k$ is under evolutionary control but all other vital rates are the same for the resident and the mutant. We therefore drop the superscripts res and mut from all other ingredients than $k$ and consider $R^{\text {mut }}$ given by (5.14) as a function of $k^{\text {res }}$ and $k^{\text {mut }}$. The ESS is obtained by maximising $R^{\text {mut }}$ in $k^{\text {mut }}$ and then putting mutant equal to resident.

We assume that $g$ and $\mu$ are continuous, that $g-\mu$ is decreasing and that the equation

$$
g(x)-\mu(x)=0
$$

has a unique positive solution, which we denote by $\tilde{x}$.

It follows from (4.4) that maximising $R^{\text {mut }}$ amounts to maximising $\Lambda_{D}^{\text {mut }(d)}(x)$ for all $x$. It follows from our assumptions that for all $x$ one has

$$
\begin{aligned}
& \Lambda_{D}^{\operatorname{mut}(d)}(x) \leq \int_{x}^{x_{\max }(D)} \frac{k^{\mathrm{mut}}(y)}{g(y) y-k^{\mathrm{res}}(y) y+\alpha D} \\
& \quad \times \exp \left(\int_{x}^{y} \frac{g(x)-k^{\mathrm{mut}}(\xi)-\mu(x)}{g(\xi) \xi-k^{\mathrm{res}}(\xi) \xi+\alpha D} d \xi\right) d y .
\end{aligned}
$$

If the mutant local population is initiated when the resident local population has size $x>\tilde{x}$, then

$$
\begin{aligned}
& \Lambda_{D}^{\operatorname{mut}(d)}(x)<\int_{x}^{x_{\max }(D)} \frac{k^{\mathrm{mut}}(y)}{g(y) y-k^{\mathrm{res}}(y) y+\alpha D} \\
\times & \exp \left(-\int_{x}^{y} \frac{k^{\mathrm{mut}}(\xi)}{g(\xi) \xi-k^{\mathrm{res}}(\xi) \xi+\alpha D} d \xi\right) d y=1 .
\end{aligned}
$$

A mutant disperser arriving at a local population with size $x>\tilde{x}$ will therefore on average produce less than one new mutant disperser. It follows that it does not pay to stay in such a population; the mutant should leave immediately. In our formulation this requires the per capita emigration rate to be infinite for $x>\tilde{x}$.

It is easily seen that if $x<\tilde{x}$, then the number of mutant dispersers produced is maximised by not producing any dispersers until $x$ reaches $\tilde{x}$ at which time all mutants in the local population should leave. In other words, the per capita emigration rate should be zero for $x<\tilde{x}$.

What happens at the critical size $x=\tilde{x}$ ? Because all individuals leave for $x>\tilde{x}$ one must have $x_{\max }(D)=\tilde{x}$. $k(\tilde{x})$ can now be solved from the equation $g(x) x-k(x)+\alpha D=0$. We have thus shown that the evolutionarily stable dispersal strategy is

$$
k(x)= \begin{cases}0 & \text { if } x<\tilde{x} \\ g(\tilde{x})-\frac{\alpha D}{\tilde{x}} & \text { if } x=\tilde{x} \\ \infty & \text { if } x>\tilde{x}\end{cases}
$$

Strictly speaking it does not make sense to model population dynamics with rates that take on infinite values on intervals of positive measure. But turning 
to the cumulative framework (now at the level of individuals as opposed to local entities as previously in this paper) this is easily remedied. Let us first note that our model is memoryless in the sense that an individual born in a patch with local population size $x$ is indistinguishable from a disperser arriving at a patch with local population size $x$. In the cumulative framework the individual dispersal strategy is most conveniently described in the context of a thought experiment in which the individual in question is equipped with a guardian angel who eliminates all causes of death (both individual death and catastrophes). The cumulative ingredient is thus

$$
\begin{aligned}
& \lambda_{D}(x ; t) \text { the probability that an individual born in (or arriving at) a patch } \\
& \text { with local population size } x \text { has not migrated } t \text { time-units later, } \\
& \text { given that the individual is not subject to any risk of death. }
\end{aligned}
$$

The content of formula (5.18) can now more appropriately be formulated by saying that the ES dispersal strategy is given by

$$
\lambda_{D}(x ; t)= \begin{cases}1 & \text { if } x<\tilde{x} \text { and } t<T_{D}(x, \tilde{x}), \\ \exp \left(-\left(g(\tilde{x})-\frac{\alpha D}{\tilde{x}}\right)\left(t-T_{D}(x, \tilde{x})\right)\right) & \text { if } x<\tilde{x} \text { and } t \geq T_{D}(x, \tilde{x}), \\ \exp \left(-\left(g(\tilde{x})-\frac{\alpha D}{\tilde{x}}\right) t\right) & \text { if } x=\tilde{x}, \quad t>0, \\ 0 & \text { if } x>\tilde{x}, \quad t>0 .\end{cases}
$$

Here $T_{D}(x, y)$ is the time it takes for a population to grow from size $x$ to size $y$. In words, at ESS an individual born in or arriving at a patch with local population size less than $\tilde{x}$ should stay until the population reaches the size $\tilde{x}$. An individual born in or arriving at a patch with local population size precisely $\tilde{x}$ should stay for an exponentially distributed time. Nobody should stay if the local population size exceeds $\tilde{x}$.

We mention in passing that the cumulative formulation of the full problem including death is quite complicated because we have to deal with a so-called competing risk problem with dependent risks. But these complications are of no importance for the ESS problem and they are therefore omitted.

Finally we observe that at the ESS the equilibrium distribution of local population sizes is a measure $p$ concentrated on $[0, \tilde{x}]$. It has an absolutely continuous part with density

$$
\phi(x)=\frac{b^{(p)}}{g(x) x+\alpha D} \exp \left(-\int_{0}^{x} \frac{\mu(\xi)}{g(\xi) \xi+\alpha D} d \xi\right)
$$

and an atom at $\tilde{x}$ containing the rest of the mass of $p$.

We close by pointing out that adding physiological structure of the individuals leaves most of the arguments in the above example intact. Only the rank of the reproduction operator becomes larger since we have to take the individual state of dispersers into account. But the fitness and ESS can be calculated as above mutatis mutandis.

\section{References}

Diekmann, O., Metz, J.A.J., and Sabelis, M.W. (1988). Mathematical models of predator-prey-plant interactions in a patchy environment, Experimental and Applied Acarology 5, 319-342. 
Diekmann, O., Metz, J.A.J., and Sabelis, M.W. (1989). Reflections and calculations on a prey-predator-patch problem, Acta Applicandae Mathematicae 14, 23-25.

Diekmann, O., Heesterbeek, J.A.P., and Metz, J.A.J.(1990). On the definition of the basic reproduction ratio $R_{0}$ in models for infectious diseases in heterogeneous populations, J. Math. Biol. 28, 365 - 382.

Diekmann,O., Gyllenberg, M., Metz, J.A.J. and Thieme, H.R. (1993). The "cumulative" formulation of (physiologically) structured population models, In "Evolution Equations, Control Theory and Biomathematics" (Ph. Clément and G. Lumer eds.), pp.145 - 154, Marcel Dekker, New York.

Diekmann, O., Gyllenberg, M., Metz, J.A.J. and Thieme, H.R. (1998). On the formulation and analysis of general deterministic structured population models: I Linear theory. J. Math. Biol. 36: 349-388.

Doebeli, M. and Ruxton, G.D. (1997). Evolution of dispersal rates in metapopulation models: branching and cyclic dynamics in phenotype space. Evolution 51, 1730-1741.

Gyllenberg, M. and Hanski, I. (1992). Single-species metapopulation dynamics: A structured model, Theor. Pop. Biol. 42, 35-62.

Gyllenberg, M. and Hanski, I. (1997). Habitat deterioration, habitat destruction and metapopulation persistence in a heterogeneous landscape, Theor. Pop. Biol. 52, 198 - 215.

Gyllenberg, M., Hanski, I. and Hastings, A. (1997). Structured metapopulation models. In I. Hanski and M. Gilpin (eds.) Metapopulation Dynamics: Ecology, Genetics and Evolution, pp. 93-122, Academic Press, London.

Hanski, I. and Gilpin, M. (1997). Metapopulation dynamics: Ecology, genetics and evolution, Academic Press, London.

Hanski, I. (1999). Metapopulation Ecology, Oxford University Press, Oxford.

Hanski, I. and Gyllenberg, M. (1993). Two general metapopulation models and the core-satellite species hypothesis, Am. Naturalist 142, 17 - 41.

Hanski, I. and Gyllenberg, M. (1997). Uniting two general patterns in the distribution of species, Science 275, 397 - 400.

Hastings, A. (1983). Can spatial variation alone lead to selection for dispersal? Theor. Pop. Biol. 24, 244-251.

Heesterbeek, J.A.P. (1992). " $R_{0}$ ", PhD Thesis, Rijksuniversiteit Leiden.

Holt, R.D. and McPeek, M.A. (1996). Chaotic population dynamics favors the evolution of dispersal, Am. Naturalist 148, 709-718.

Metz, J.A.J. and Diekmann, O. (1986). The Dynamics of Physiologically Structured Populations. Lecture Notes in Biomathematics 68. Springer, Berlin.

Metz, J.A.J. and Gyllenberg, M. (1999). How should we define fitness in structured metapopulation models? Including an application to the calculation of ES dispersal strategies. Research Report A39, University of Turku, Institute of Applied Mathematics. 
Metz, J.A.J., Nisbet, R.M. and Geritz, S.A.H. (1992). How should we define "fitness" for general ecological scenarios? TREE 7: 198-202.

Parvinen, K. (1999). Evolution of migration in a metapopulation, Bull. Math. Biol. 61, 531-550. 